\title{
Experimental Presentation of Microwave Absorption due to Shaking of JV by AC Magnetic Field in Bi2212 and Bi2223
}

\author{
D. Shaltiel $\cdot$ H.-A. Krug von Nidda $\cdot$ B.Y. Shapiro • \\ B. Rosenstein • A. Loidl • B. Bogoslavsky • I. Shapiro • \\ T. Fujii · T. Watanabe · T. Tamegai
}

Received: 16 November 2008 / Accepted: 21 November 2008 / Published online: 4 December 2008

(C) The Author(s) 2008. This article is published with open access at Springerlink.com

\begin{abstract}
The Josephson Vortex dynamics in high anisotropy superconductors Bi2212 and Bi2223 induced by AC magnetic field collinear to DC magnetic field and parallel to the layers is studied via their interaction with microwave field. Experimental results as function of DC magnetic field, AC magnetic field and temperature are presented. The AC induced microwave dissipation is larger than dissipation without AC field. The results are explained by the theoretical interpretation reported recently due to shaking by the AC field that depins the JV (Shaltiel et al., Phys. Rev. B 77, 014508, 2008). Similar behavior in these two compounds as a function of the variables involved discloses that
\end{abstract}

D. Shaltiel $(\bowtie) \cdot$ B. Bogoslavsky

Racah Institute of Physics, The Hebrew University of Jerusalem, Jerusalem, Israel

e-mail: shaltiel@vms.huji.ac.il

H.-A. Krug von Nidda $\cdot$ A. Loidl

Experimental Physics V, Center for Electronic Correlations and

Magnetism, University of Augsburg, 86135 Augsburg, Germany

B.Y. Shapiro · I. Shapiro

Physics Department, Bar-Ilan University, Ramat-Gan, Israel

B. Rosenstein

Department of Electrophysics, National Chiao Tung University, Hsinchu, Taiwan

T. Fujii

Cryogenic Center, The University of Tokyo, Tokyo 113, Japan

T. Watanabe

School of Science and Technology, Hirosaki University, Hirosaki, Aomori 033-8561, Japan

T. Tamegai

Electrophysics Department, The University of Hongo, Tokyo

113-8656, Japan the shaking effect should be observed in any high anisotropy superconductors. It shows that the AC field interacting with $\mathrm{JV}$ has an active and not the usual passive modulating role of the DC field in EPR dissipation experiments. The technique can be used to investigate JV dynamics and JV phase diagram.

Keywords High anisotropy superconductors $\cdot$ Bi-based cuprates - Josephson vortices and fluxons · Response to microwave and to magnetic field in superconductors . Superconductivity phase diagram

\section{Introduction}

Investigation of Josephson sine-Gordon solitons, known as fluxons, has drawn great interest in basic and applied physics [2]. Most investigations are performed in mesastructures of intrinsic Josephson junction IJJ stacks of superconducting layers coupled by Josephson effect. The linear size of sacks is usually less than $100 \mu \mathrm{m}$, where the Josephson solitons move along its junction [3]. The purpose of those investigations is to study the dynamic behavior of fluxons where emphasis is drawn on the collective motion of the Josephson vortices generated by electrical current and detected via the I-V curves [4]. The investigations are motivated for the possible application to generate radiation at THz frequencies [5].

In the present work the experimental method of "Induced Microwave Dissipation by AC Magnetic Field" (IMDACMF) [6] is introduced to study the dynamic behavior of solitons in bulk high-anisotropy single superconductor crystals of Bi2212 and Bi2223, via their interaction with the magnetic component of the microwave field. The interaction is attained by applying an AC magnetic field collinear 
with a DC magnetic field parallel to the $a-b$ plane. The AC field varies the density of the fluxons due to their motion in and out of the superconductor, causing interaction with the microwave within its skin depth. The interaction induces a microwave dissipation caused by the collective motion of the JV; we show that the dissipation is by far larger than the dissipation due to the interaction of the microwave with the JV without the presence of the AC magnetic field [7]. Differences in the microwave power dissipation of more than two orders of magnitude were observed with or without the presence of AC field in Bi2212 crystals where a very similar experimental setup was applied. It indicates that the AC field strongly enhances the microwave dissipation in highanisotropy superconductors that results from the interaction of the JV with microwave magnetic field. The results indicate that, depending on the variables involved, the microwave response can be linear or nonlinear with the $\mathrm{AC}$ field. Furthermore, the signal phase with respect to the external $\mathrm{AC}$ phase can vary with the change in the variables involved.

Based on the present experimental results, the ACinduced microwave dissipation was recently interpreted theoretically in the framework of Josephson electrodynamics in layered superconductors [1]. It shows that the enhancement of the microwave dissipation in the presence of $\mathrm{AC}$ field results from the shaking of JV by the AC field.

The IMDACMF technique introduces a new method to investigate the dynamics of fluxons. It is different from the IJJ method mentioned above. In the IJJ method the motion of the JV vortices is obtained by applying electric current perpendicular to the conducting planes of the superconductor and its effect is observed by DC resistivity and I-V characteristics measurements. The present method is contactless; the motion of fluxons is induced by an AC field collinear to the DC field and parallel to the conduction planes, and its effect is detected via microwave dissipation. It can be applied to investigate the effect of different treatments of the superconductor on the motion of the fluxons. A greater variety of samples, small and large single crystals, can be used with this technique.

It should be mentioned that after the discovery of high $T_{\mathrm{c}}$ superconductors, EPR spectrometers where extensively used to investigate their interaction with microwave [8]. Though interesting results where obtained, the technique was soon almost abandoned as it did not reveal additional substantial information. The present work shows a new experimental procedure to obtain results on the interaction of vortices with the microwave in high-anisotropy superconductors, using EPR spectrometers.

In the following we describe the experimental set up and the measuring procedure. We then present the experimental results of the AC-induced microwave dissipation in the high anisotropy Bi2212 and Bi2223 crystals, as a function of the variables involved, namely, DC magnetic field, AC magnetic field and temperature, where similarities and differences in their behavior are exposed. The next section analyzes the experimental results. Then the experimental results are compared with the theoretical work [1]; it shows a good qualitative agreement with the experimental results. The discussion summarizes the important results and points out new possibilities to investigate the dynamics of JV by the IMDACMF technique.

\section{Experimental}

\subsection{Experimental Setup}

The experiment block diagram is shown in Fig. 1. It consists of a Bruker ELEXSYS continuous EPR spectrometer working at $\mathrm{X}$-band frequency $(9.36 \mathrm{GHz})$ and a digital oscilloscope. A microwave source feeds a cavity where highanisotropy Bi2212 or Bi2223 crystals $\left(1 \times 1 \times 0.1 \mathrm{~mm}^{3}\right)$ are placed in the cavity region where only the microwave magnetic field is present. The sample, whose temperature could be varied down to helium temperature, was exposed to a collinear DC and $\mathrm{AC}(100 \mathrm{~Hz}$ to $100 \mathrm{kHz})$ magnetic fields. The microwave magnetic field was parallel to the $a-b$ plane and perpendicular to the DC magnetic field. The AC magnetic field induces a signal into the microwave power reflected from the cavity. It is rectified by a diode that feeds either a lock-in detector or a digital oscilloscope. The lock-in gives the signal intensity and phase of the first AC harmonic; the oscilloscope displays also higher harmonics within its band width. The combined detectors present direct information of the microwave dissipation at the AC frequency. Unless otherwise specified, the magnetic fields were parallel to the $a-b$ plane where only JV are formed in contrast to magnetic field parallel to the $c$-axis where only PV are formed (Fig. 2). The experiments were performed using the ZFC procedure, namely, the sample was cooled at zero magnetic field from above $T_{\mathrm{c}}$ to the required temperature. The $\mathrm{AC}$ or DC field was then set to a predetermined value and the $\mathrm{DC}$ or the AC magnetic field respectively was increased in steps. The signal was registered at each field. The reason for increasing the fields in steps and not continuously is that not only the signal intensity but also the $\mathrm{AC}$ frequency signal phase varied as a function of the variables, as is demonstrated in Fig. 3. Thus when measuring with the lock-in detector it was necessary to determine the phase at maximum signal for each measurement. Here the phase values are stated with respect of the phase of the external AC field $h_{\mathrm{AC}}$. Analyzing the results obtained from the lock-in and from the digital oscilloscope gave a full picture of the signal response, including signal intensity, signal shape and 
Fig. 1 Block diagram of the experimental setup

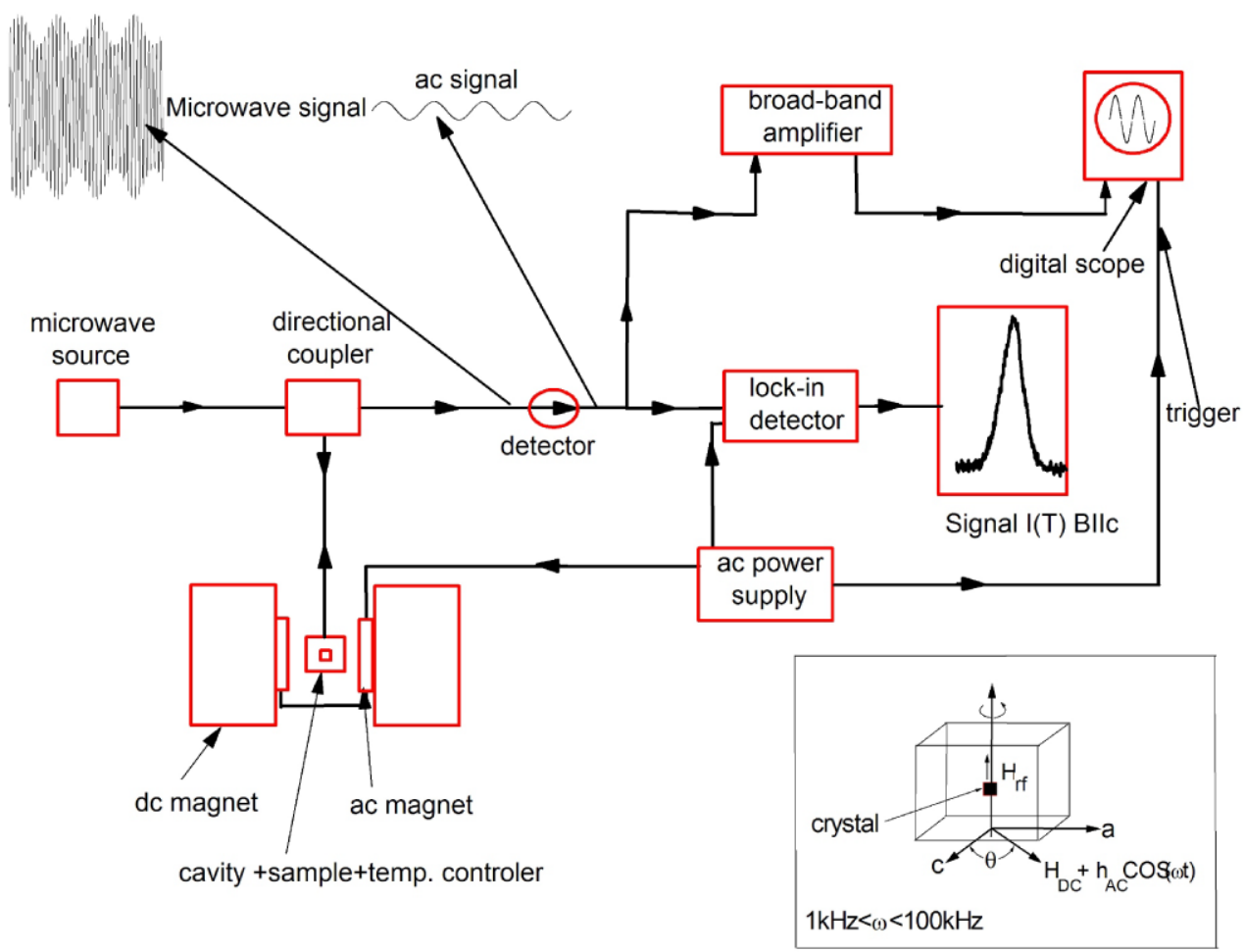

signal phase. The method of aligning the crystal can be deduced from a previous work [9]. It is performed by rotating the crystal around an axis parallel to the $c$-axis [6, Fig. 2].

Two high-anisotropy compounds were investigated, an optimally doped Bi2212 crystal $T_{\mathrm{c}}=90 \mathrm{~K}$ and several Bi2223 crystals whose $T_{\mathrm{c}}$ varied between $110 \mathrm{~K}$ and $105 \mathrm{~K}$. First, the experimental results of the Bi2212 are presented, followed by the $\mathrm{Bi} 2223$ results.

\subsection{Experimental Results of Bi2212}

Figure 3 shows the oscilloscope signals of a Bi2212 crystal for the same AC field at two different DC fields (the upper frame), and for the same DC field at two different AC fields (the lower frame). The figure indicates that the signal can have a sinusoidal or a non-sinusoidal shape indicating linear or nonlinear response, respectively, for the same AC field or for the same DC field. The figure also shows that the AC phase of the fundamental harmonics depends on the intensities of the AC or DC field. Additional measurements have shown that the phase may depend also on the magnitude of other variables, such as temperature.

Figure 4 shows signal intensity as a function of temperature from slightly above $T_{\mathrm{c}}$ down to $4 \mathrm{~K}$ for magnetic field, with $H_{\mathrm{DC}}$ parallel to the c direction or parallel to the $a-b$ plane. At magnetic field parallel to the $c$-axis, where only $\mathrm{PV}$ are formed (Fig. 2), the signal intensity down to $4 \mathrm{~K}$ is zero, except the narrow range signal just below $T_{\mathrm{c}}$. It results from the variation of the thermal activated flux flow resistivity [10] (TAFF), and is reported in detail in reference [6]; it will not be discussed further. For $H_{\mathrm{DC}}$ parallel to the $a-b$ plane where only JV are formed (Fig. 2) the signal intensity increases sharply at $T_{\mathrm{c}}$ and even further down to $4 \mathrm{~K}$. The results in Fig. 4 indicate that the AC magnetic field induces strong microwave dissipation when interacting with JV but almost none when interacting with PV.

The lock-in signal intensity and signal phase as a function of DC fields at $40 \mathrm{~K}$ at a low-AC field and a highAC field of $0.1 \mathrm{mT}$ and $1.2 \mathrm{mT}$, respectively, are shown in Fig. 5a. Different functional behavior is observed at these two AC fields. At the low-AC field the signal intensity below $H_{\mathrm{DC}}=4 \mathrm{mT}$ is small. An asymmetric bell-shaped curve is observed at higher fields, with a stronger slope in the low field side and a maximum at $13 \mathrm{mT}$. The signal phase is close to $-180^{\circ}$ in the whole field region. The high-AC field signal intensity has a maximum at $2 \mathrm{mT}$, the intensity which is more than three orders of magnitude larger than the intensity of the low-AC field signal with the same DC field. This maximum decreases strongly, followed by a second broader maximum at $19 \mathrm{mT}$. The signal phase associated with the first maximum is around $-30^{\circ}$ : it decreases sharply at higher fields and tends asymptotically to $-160^{\circ}$ at the highest DC field.

The digital oscilloscope signals along the DC field for the low-AC field and the high-AC intensity curves of Fig. 5a are shown in Figs. 5b and 5c respectively. Figure 5b shows that for $h_{\mathrm{AC}}=0.1 \mathrm{mT}$ the signal intensity at the two low-DC fields is weak and its signal shape deviates strongly from the sine, indicating a nonlinear response. The intensity increases 

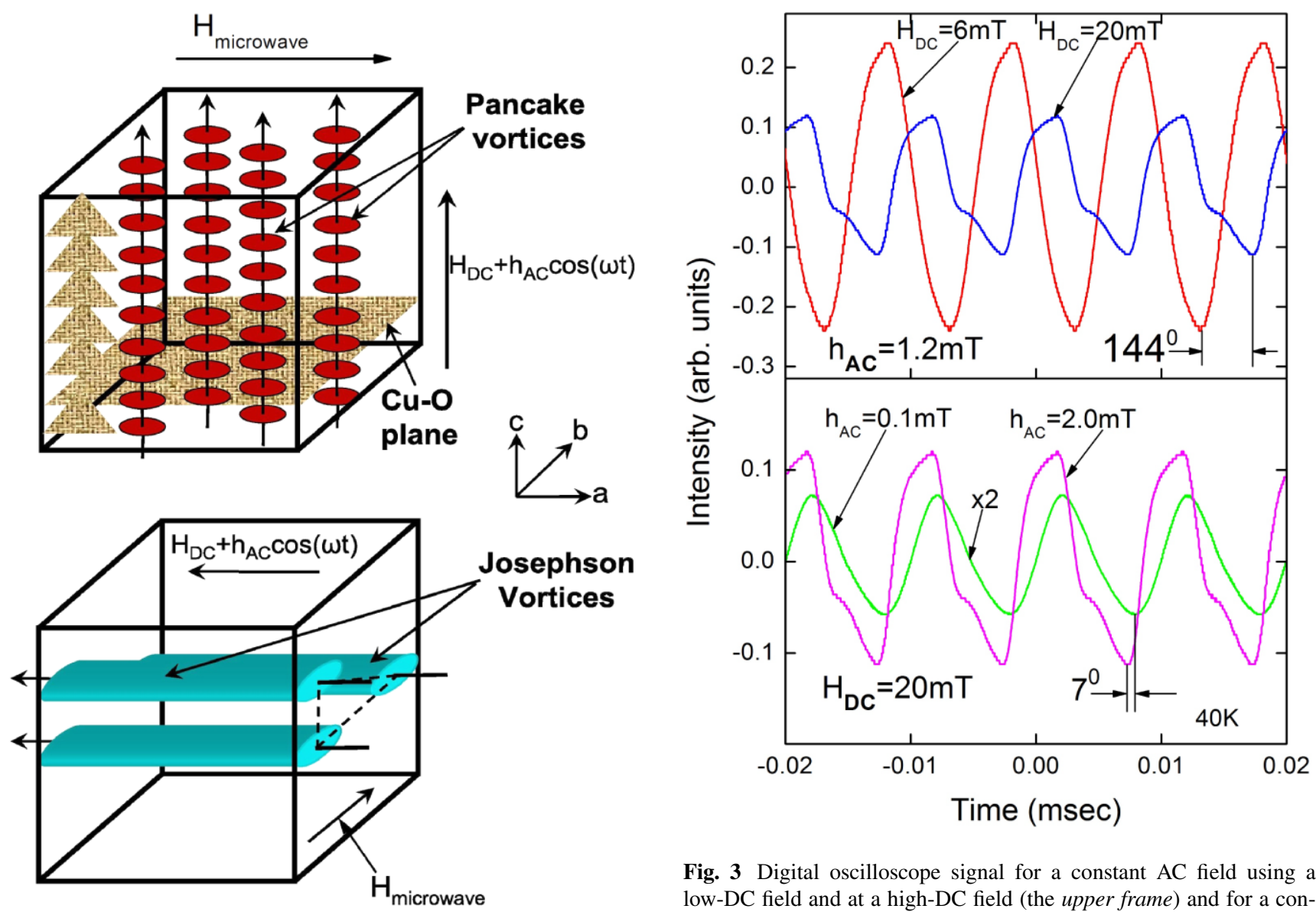

Fig. 3 Digital oscilloscope signal for a constant AC field using a low-DC field and at a high-DC field (the upper frame) and for a constant DC field using a low-AC field and a high-AC field (the lower frame)

2 Vortex structures in high-anisotropy superconductors for netic field $H_{\mathrm{DC}} \| c$-axis where only $\mathrm{PV}$ are formed and $H_{\mathrm{DC}} \| a-b$ plane where only JV are formed

strongly at higher DC fields and its signal shape is close to sine, indicating a linear response. Figure $5 \mathrm{c}$ shows that the signal shape for $h_{\mathrm{AC}}=1.2 \mathrm{mT}$ at the magnetic field close to the first maximum is close to sine, indicating a linear response. It starts to deviate from the sine already at $10 \mathrm{mT}$ with maximum deviation from the sine at $15 \mathrm{mT}$. At higher fields the deviation from sine shape decreases with increasing field. Thus the signal shape in Figs. $5 \mathrm{~b}$ and $5 \mathrm{c}$ clarifies the changes in the signal intensities and phases as a function of DC field, as was shown in Fig. 4a.

Figure 6a shows the lock-in signal intensity and phase as a function of AC fields at $40 \mathrm{~K}$ at a low-DC field of $2.5 \mathrm{mT}$ and at a high-DC field of $22 \mathrm{mT}$. The two DC fields are close to the two maxima shown in Fig. 5 for $h_{\mathrm{AC}}=1.2 \mathrm{mT}$. Different behavior is observed at these two curves. At the low-DC field's maximum curve, the lock-in signal intensity is close to zero below a threshold AC field. Above this threshold the microwave dissipation increases linearly. At the high-DC field's maximum curve the signal intensity increases linearly from zero up to about $h_{\mathrm{AC}}=0.5 \mathrm{mT}$, then it saturates strongly up to the highest $\mathrm{AC}$ field. The phase

of the low-DC field signal is close to zero $\left(-25^{\circ}\right.$ on average). The phase of the high-DC signal is equal to $-180^{\circ}$ at low-AC fields and tends asymptotically to $-140^{\circ}$ at the highest AC field. The digital oscilloscope signals along the curves of the low-DC field maximum and the high-DC maximum are shown in Figs. $6 \mathrm{~b}$ and $6 \mathrm{c}$ respectively. Figure $6 \mathrm{~b}$ shows that at $h_{\mathrm{AC}}=0.1 \mathrm{mT}$ the low-DC signal intensity is weak and its signal shape deviates strongly from sine, indicating a nonlinear response. The intensity increases strongly at higher AC fields and its signal shape is close to the sine, indicating a linear response. Figure $6 \mathrm{c}$ shows that the highDC field signal shape at $h_{\mathrm{AC}}=0.1 \mathrm{mT}$ is the sine indicating a linear response. At higher AC field the signal shape deviates from the sine indicating a nonlinear response. Thus the signal shape in Fig. $6 \mathrm{~b}$ and Fig. $6 \mathrm{c}$ clarifies the changes in the signal intensities and phases as a function of $\mathrm{AC}$ field shown in Fig. 6a.

Figure 7 presents the temperature evolution of the signal intensity as a function of DC field from $80 \mathrm{~K}$ to $4 \mathrm{~K}$ for the "low-AC" field (0.1 mT) and the "high-AC" field (1.2 mT). It shows that the intensity increases with decreasing temperature as also is indicated in the insets of the figure. 


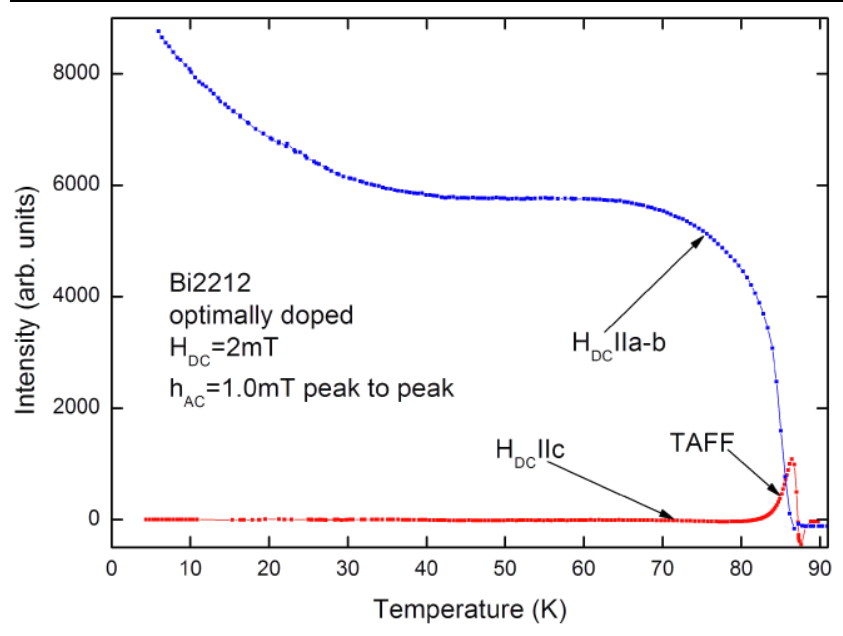

Fig. 4 Intensity as a function of temperature when applying magnetic field parallel and perpendicular to the conduction planes. At $H \| a-b$ a strong signal is observed from $T_{\mathrm{c}}$ down to $4 \mathrm{~K}$. The intensity as a function of temperature for $H \| c$ is zero except for the FFR signal just below $T_{\mathrm{c}}$

\subsection{Dependence of Microwave Dissipation on the Microwave Power}

The applied microwave magnetic field in the present work was $0.007 \mathrm{mT}(6.3 \mathrm{~mW}$ in a cavity with $Q=3300)$. The interaction of the $\mathrm{JV}$ with the microwave for different microwave power, Fig. 8, indicates that the signal intensity for the same AC and DC fields is proportional to the square root of the microwave power implying an ohmic behavior. Similar ohmic behavior was observed by Enriquez [11] for the same experimental setup but without the application of the AC field.

The unusual high intensity response of microwave dissipation by AC magnetic field in Bi2212 was not observed in the low-anisotropy optimally doped $\mathrm{YBaCuO}_{7}$ superconductor with magnetic fields parallel to the $a-b$ plane. A fundamental difference between these two compounds regarding Josephson vortices is that in the high-anisotropy Bi2212 the coupling mechanism between the layers is due to the Josephson effect. To find out if this dissipation is related to the JV inter-layered coupling, we have extended the investigated to Bi2223. Though its anisotropy is slightly lower than that of $\mathrm{Bi} 2212$ [12], the coupling mechanism between the layers is due to the JV.

\subsection{Experimental Results of $\mathrm{Bi} 2223$}

Several Bi2223 crystals were investigated whose $T_{\mathrm{c}}$ ranges from $105 \mathrm{~K}$ to $111 \mathrm{~K}$. Here we present the results of a $T_{\mathrm{c}}=$ $105 \mathrm{~K}$ crystal the transition temperature of which though was slightly lower than in other crystals, the results show a clearer behavior attributed to smaller number of defects [13].
Figure 9a shows the signal intensity as a function of temperature for $H \| a-b$ and $H \| c$, with $H_{\mathrm{DC}}=1 \mathrm{mT}$ and $h_{\mathrm{AC}}=0.4 \mathrm{mT}$. At $H \| c$ the signal just below $T_{\mathrm{c}}$ results from the TAFF resistivity discussed earlier. A strong anomalous signal with a maximum at $84.5 \mathrm{~K}$ and a width of $4 \mathrm{~K}$ is observed. The signal intensity below $85 \mathrm{~K}$ is practically zero. At $H \| a-b$ the signal intensity increases slightly and gradually down to around $88 \mathrm{~K}$, where a strong anomalous signal is observed at $83 \mathrm{~K}$, similar in shape and intensity to the anomalous signal observed for $H \| c$. Below the anomalous signal, a relatively-high intensity signal is observed down to $4 \mathrm{~K}$. At high-anisotropy superconductors the TAFF signal intensity for $H \| a-b$ is almost zero [6]. The small TAFF signal observed at this orientation in Fig. 9a results from a slight deviation of the magnetic field (about $3^{\circ}$ away the $a-b$ plane). Indeed, when aligning the crystal almost parallel to the $a-b$ plane, the small TAFF signal just below $T_{\mathrm{c}}$ is not observed (Fig. 9b). Figure 10 shows the lock-in signal as a function of DC field at $4 \mathrm{~K}$ for a low-AC and a highAC field of $0.1 \mathrm{mT}$ and $1.2 \mathrm{mT}$, respectively. The low-AC field signal intensity has an asymmetric bell-shaped curve with a smaller slope at the high field side with the maximum at $8 \mathrm{mT}$. The high-AC field signal intensity has two maxima. The maximum of the low field signal is at $1 \mathrm{mT}$ and its intensity is more than 2 orders of magnitude stronger than the signal intensity of the low-AC field intensity signal at the same DC field. The intensity below this maximum decreases sharply with the field and then increases towards a second maximum. Above the second maximum, the intensity decreases gradually towards zero at $100 \mathrm{mT}$. The signal phase of the low-AC field is about $-180^{\circ}$. The signal phase of the high-AC field has a maximum of $-60^{\circ}$ followed by a sharp decrease that tends asymptotically to $-160^{\circ}$ at fields higher than $100 \mathrm{mT}$.

Figure 11a shows the lock-in signal intensity and phase at $20 \mathrm{~K}$ as a function of AC field for a low-DC field and a highDC field of $6 \mathrm{mT}$ and $20 \mathrm{mT}$, respectively. The low and high DC field intensities were chosen to be close to the maxima observed in Fig. 10. At the low-DC field the signal intensity above a threshold AC field increases almost linearly up to about $h_{\mathrm{AC}}=2 \mathrm{mT}$ and then it starts to saturate. The signal intensity at the high-DC field increases linearly already from zero but saturates already at $h_{\mathrm{AC}}=0.6 \mathrm{mT}$. The phase of the low-DC field is close to $-180^{\circ}$ at $\mathrm{AC}$ field of $0.1 \mathrm{mT}$ and increases sharply at higher AC field. The phase of the high-DC field signal has an almost constant value of $-170^{\circ}$ up to $h_{\mathrm{AC}}=0.3 \mathrm{mT}$ and increases gradually to $-130^{\circ}$ at the highest $h_{\mathrm{AC}}$ field of $3 \mathrm{mT}$. The digital oscilloscope signals along the two curves of Fig. 11a are shown in Figs. 11b and 11c. They clarify the strong changes in the intensity and phase as a function of AC field observed in the two curves of Fig. 11a. 


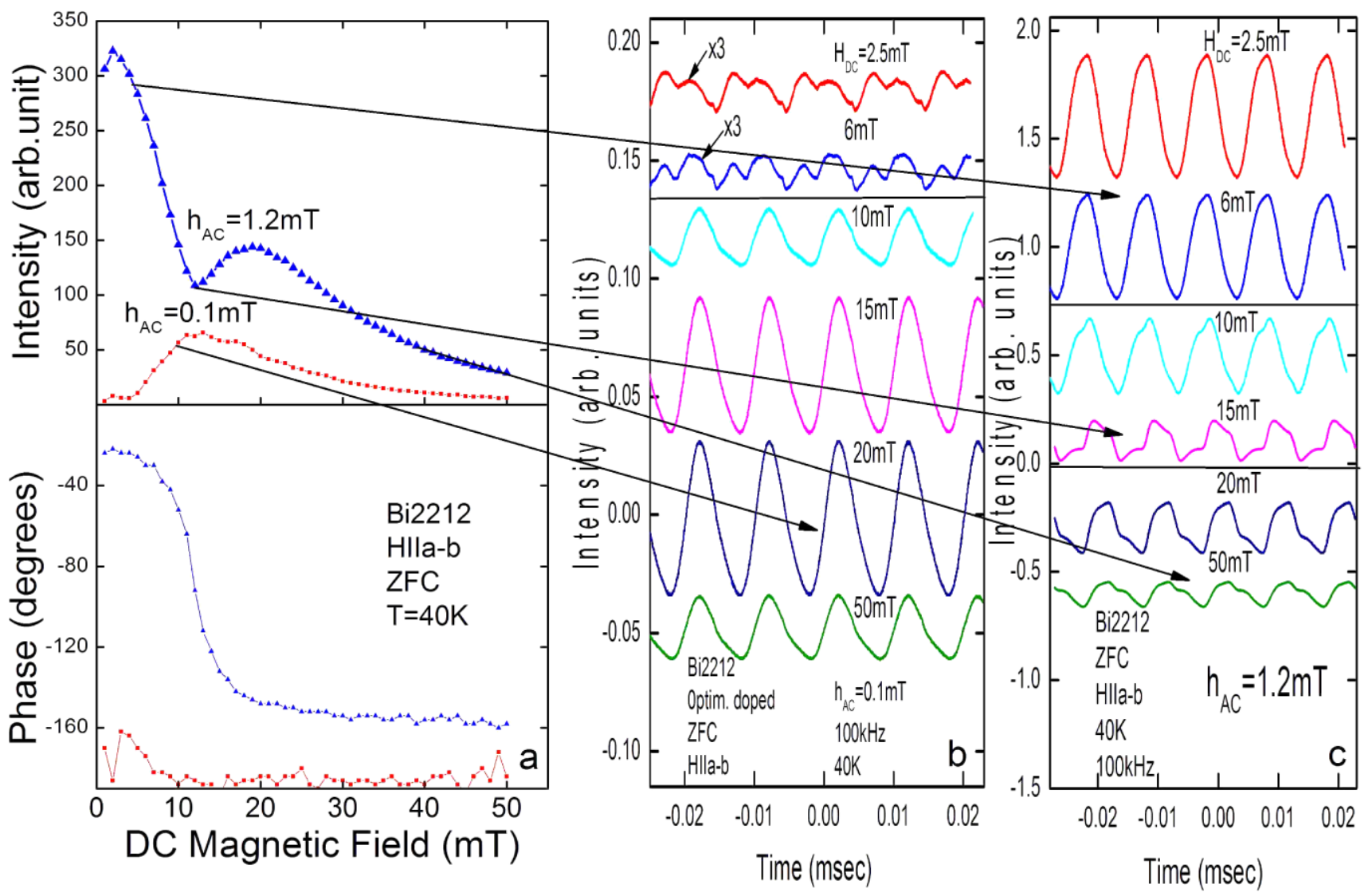

Fig. 5 (a) Lock-in signal intensity and phase as a function of DC fields at $40 \mathrm{~K}$ at a low-AC field and at high-AC field of $0.1 \mathrm{mT}$ and $1.2 \mathrm{mT}$ respectively. (b) Oscilloscope signal along the curve of the

The evolution of the signal intensity as a function of DC field from $90 \mathrm{~K}$ to $4 \mathrm{~K}$ for a low-AC field of $0.3 \mathrm{mT}$ and a high-AC field of $1.2 \mathrm{mT}$ is shown in Fig. 12.

\section{Analysis of the Experimental Results}

\section{$3.1 \operatorname{Bi} 2212$}

Figure 4 shows that the AC field collinear with the DC field induces microwave dissipation as a function of temperature down to $4 \mathrm{~K}$ for magnetic field parallel to the $\mathrm{Cu}$ planes, where only JV are formed. There is no microwave dissipation for magnetic field parallel to the $c$-axis where only PV are formed. It leads to the fundamental conclusion that the induced microwave dissipation results from the AC interaction with JV but not with PV. The increase of the signal intensity with decreasing temperature for $H \| a-b$ (Fig. 4) signifies that the dissipation does not result from a static property, as decrease in the absorbed microwave power with decreasing temperature is expected due to pinning. Thus the observed signals result from dynamic interaction of the
low-AC field shown in (a). (c) Oscilloscope signal along the curve of the high-AC field shown in (a)

moving fluxons with the microwave field, within the superconducting microwave skin depth, whose motion is induced by the AC field. Figure 3 shows that the time dependent of the microwave dissipation depends strongly on the variables involved, such as DC magnetic field and AC magnetic field. Small changes in one parameter may change the signal shape from sinusoidal to non-sinusoidal and vary the phase more than $90^{\circ}$. These changes are related to the variation of the signal phase and shape as a function of DC and AC fields shown in Figs. 5 and 6 respectively.

The properties of this dynamic interaction as a function of the DC and AC magnetic fields at $40 \mathrm{~K}$ are manifested in the signal intensity and phase in Fig. 5a and Fig. 6a, and their corresponding signal shapes are displayed in Figs. 5b$5 \mathrm{c}$ and $6 \mathrm{~b}-6 \mathrm{c}$, respectively.

The lock-in signal intensities and their corresponding phases at $40 \mathrm{~K}$ as a function of DC field (Fig. 5), indicate that the functional behavior at the $0.1 \mathrm{mT}$ low-AC field differs substantially from the $1.2 \mathrm{mT}$ high-AC field. It confirms the conclusion obtained from Fig. 3 that the AC-induced interaction with the fluxons depends strongly on the values of the variables involved. The low-AC field curve has an asym- 


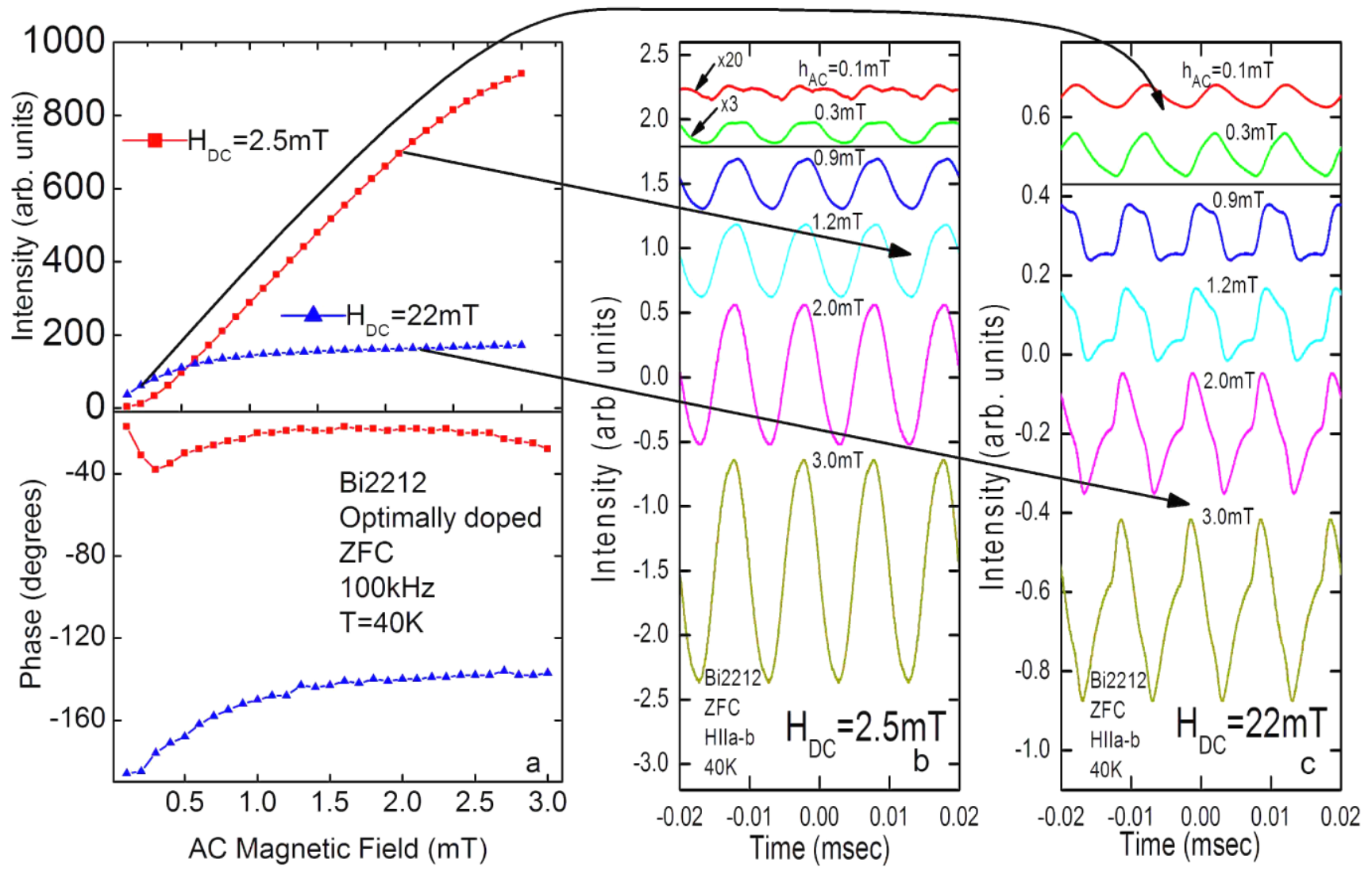

Fig. 6 (a) Lock-in signal intensity and phase in Bi2212 as a function of AC fields at $40 \mathrm{~K}$ at $H_{\mathrm{DC}}=2.5 \mathrm{mT}$ and $H_{\mathrm{DC}}=22 \mathrm{mT}$, corresponding to the two DC fields where the respective maxima of the signal intensity as function of DC shown in Fig. 5 were observed. (b) Oscilloscope signal along the curve for DC field of $2.5 \mathrm{mT}$ shown in (a). (c) Oscilloscope signal along the curve for DC field of $22 \mathrm{mT}$ shown in (a) metric bell-shaped signal whose phase is close to $-180^{\circ}$ (Fig. 5a). Its signal shape is sinusoidal indicating a linear response (Fig. 5b). Thus linear response occurs at a phase values close to $-180^{\circ}$ at low-AC field. The high-AC field curve shows two maxima, at a low-DC field of $2 \mathrm{mT}$ and at a high-DC field of $15 \mathrm{mT}$ (Fig. 5a). The intensity of the lowfield maxima is by far larger than the intensity at the low-AC field for the same DC fields. The phase of this low-field maximum is close to zero (Fig. 5a) and its line shape (Fig. 5c) is close to the sine, indicating that for phase close to zero a linear response is observed. At magnetic fields above this maximum, there is a strong decrease of the signal intensity followed by a broad maximum. The phase decreases rather sharply with the decrease of the intensity at higher DC fields and tends asymptotically toward $-160^{\circ}$. The line shape in this field range deviates from sinusoidal shape (Fig. 5c) indicating a nonlinear response. Thus Fig. 5 shows that the signal intensity as a function of DC field is strongly affected when exposed to low-AC or to high-AC field.

A clearer behavior of the effect of the AC field on the microwave dissipation is obtained from the signal intensities as a function of AC field for a $2.5 \mathrm{mT}$ at the low-DC field and $22 \mathrm{mT}$ at a high-DC field, where the two DC fields cor- respond to the position at the low and at the high DC field maxima with $h_{\mathrm{AC}}=1.2 \mathrm{mT}$ of Fig. 5. Strong differences were observed in the signal intensities and signal phases as a function of AC field at $40 \mathrm{~K}$ at these two DC fields (Fig. 6). It implies again that the $\mathrm{AC}$ interaction with the solitons strongly depends on the values of the variables involved. There is a sharp contrast in the functional dependence at the two fields: The low-DC field signal intensity is very small: up to $h_{\mathrm{AC}}=0.3 \mathrm{mT}$ it increases linearly up to $h_{\mathrm{AC}}=2.5 \mathrm{mT}$. The high-DC field signal increases linearly only up to $h_{\mathrm{AC}} \approx 0.3 \mathrm{mT}$ and remains almost constant up to $h_{\mathrm{AC}}=3 \mathrm{mT}$ (Fig. 6a). The signal phase value of the low-DC is close to zero. The high-DC signal phase is close to $-180^{\circ}$ up to $h_{\mathrm{AC}} \approx 0.3 \mathrm{mT}$ then it increases with AC field, saturating at $-140^{\circ}$ at the maximum $\mathrm{AC}$ value of $3 \mathrm{mT}$ (Fig. 6a). The signal shape of the low-DC field signal is sinusoidal indicating a linear response in the whole $\mathrm{AC}$ field range where the phase is close to zero (Fig. 6b), while the signal shape of the high-DC field is sinusoidal only up to $h_{\mathrm{AC}}=0.3 \mathrm{mT}$ where the phase is close to $-180^{\circ}$, and deviates from the sine shape above this value (Fig. 6c).

The phase behavior as a function of DC field is similar to the phase behavior as a function of AC field. In both cases, 


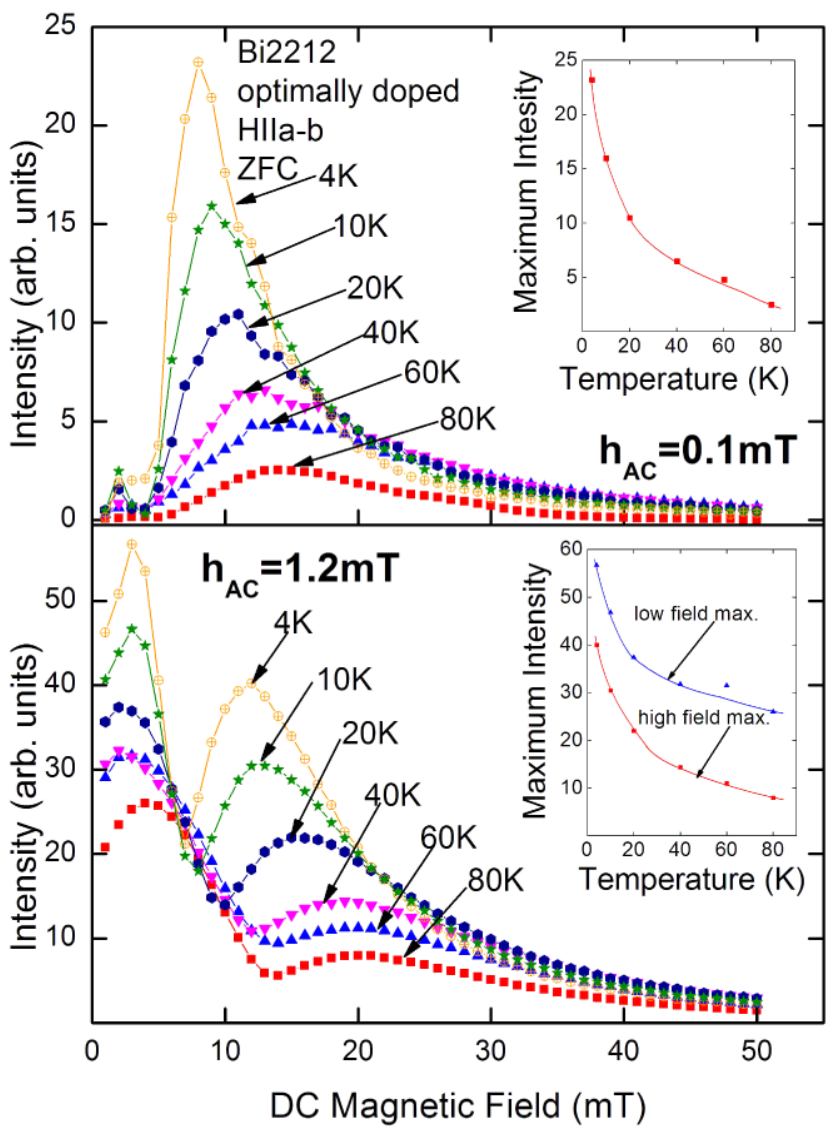

Fig. 7 Temperature evolution of the signal intensity as a function of a DC field from $80 \mathrm{~K}$ to $4 \mathrm{~K}$ for a low-AC field $(0.1 \mathrm{mT})$ and a high-AC field $(1.2 \mathrm{mT})$. It shows that the intensity increases with decreasing temperature as also indicated in the insets

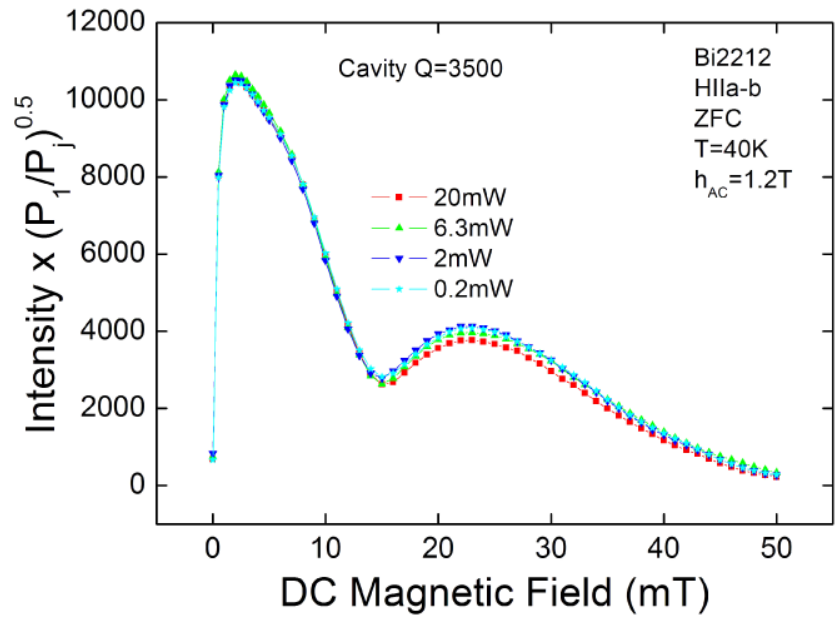

Fig. 8 Normalized signal intensities as a function of DC magnetic field, for microwave power difference of up to two orders in magnitude

a linear response is observed at phase values either close to $0^{\circ}$ or to $180^{\circ}$. Nonlinear response is observed in the narrow

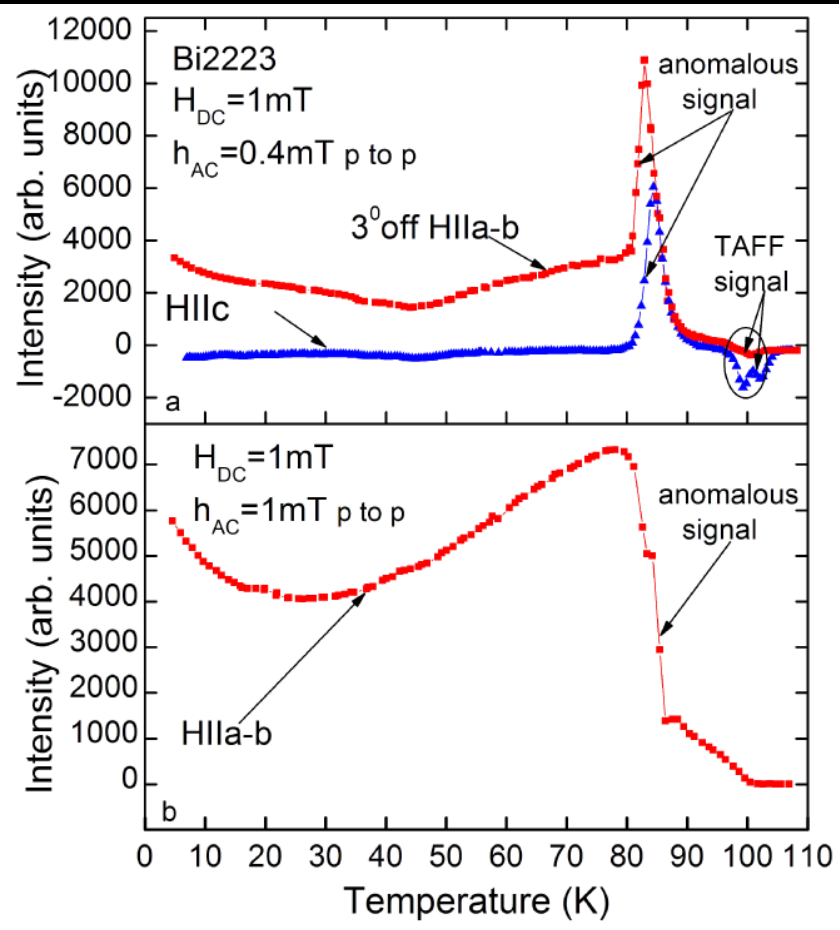

Fig. 9 (a) Signal intensity as a function of temperature in Bi2223 for magnetic fields: for $H$ almost parallel to the $a-b$ plane ( 30 off) and for $H$ parallel to the $c$-axis. The strong signals at $85 \mathrm{~K}$ for both orientations represent an anomalous signal. The small signals just below $T_{\mathrm{c}}$ result from TAFF resistivity. (b) Signal intensity as a function of temperature in Bi2223 for $H$ parallel to the $a-b$ plane. The absence of the small TAFF resistivity signal just below indicates that it is aligned almost parallel to the conduction planes

region where the phase values shift from close to $0^{\circ}$ toward $180^{\circ}$.

\subsection{Comparison between Bi2212 and Bi2223}

Induced microwave dissipation by $\mathrm{AC}$ field observed in Bi2212, where the layered superconductors are coupled via the Josephson effect, is not observed in optimally doped YBCO where there is no Josephson coupling between the layers. We deduce that coupling between the conducting layers by $\mathrm{JV}$ is a necessary condition to induce the microwave dissipation. If this assumption is correct the AC field should induce microwave dissipation in Bi2223 similar to that observed in Bi2212 as a function of any of the variables, namely DC magnetic field, AC magnetic field and temperature should show similar behavior. Indeed, the analysis presented below of the experimental results in the two compounds displays very similar behavior.

(a) Signal as a function of temperature, Figs. 4 and 9.

The signal intensity in both compounds as a function of temperature at magnetic fields parallel to the $a-b$ plane shows strong signal intensities from $85 \mathrm{~K}$ down to $4 \mathrm{~K}$ with similar behavior. At magnetic fields parallel to the $c$-axis, a 


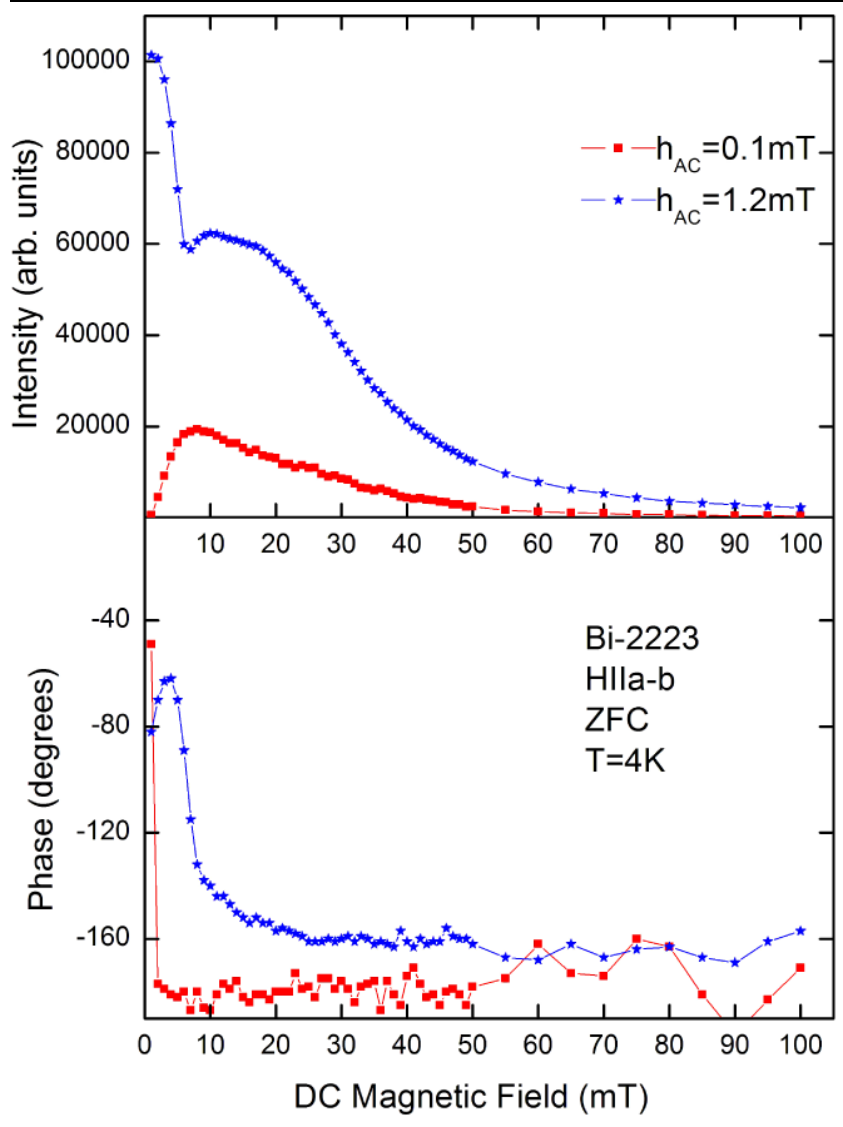

Fig. 10 Lock-in signal intensity and phase as a function of DC fields at $40 \mathrm{~K}$ at a "low-AC field" and a "high-AC field" of $0.1 \mathrm{mT}$ and $1.2 \mathrm{mT}$, respectively

FFR signal is observed just below $T_{\mathrm{c}}$ with zero signal intensity at lower temperatures.

(b) Signal as a function of DC magnetic fields at a constant temperature, Figs. 5 and 9.

Applying a low-AC field in the two compounds induces a similar asymmetric bell-shaped signal with a signal phase of $-180^{\circ}$. Two maxima are observed when exposed to a highAC field. The intensity at the lower DC field maximum is strong and its width is narrower compared to the width of the higher DC field maximum. The phase at high-AC field is closer to zero for the first maximum, and it decreases sharply with DC field tending asymptotically to $-160^{\circ}$ at higher DC field.

(c) Signal as a function of AC magnetic field at a constant temperature, Figs. 4 and 10.

In both compounds the signal intensities as function of AC field for the low-DC field (above a threshold value) increases linearly with the AC field. For the "high-DC" field the intensity increases linearly already from zero AC field and saturates above a small value of the AC magnetic field. Their corresponding phases at low and high DC fields show similar behavior (Figs. 4a and 8a). Comparison of the digital oscilloscope signals in Bi2212 (Figs. 4b and 4c) with those in Bi2223 (Figs. 10b and 10c), shows similar linear and nonlinear response at low and high intensity of the AC field.

(d) Variation of the signal intensity for temperatures $80 \mathrm{~K}$ to $4 \mathrm{~K}$ as a function of DC magnetic field at low-AC and at high-AC magnetic field, Figs. 7 and 11.

The signal intensities increase with decreasing temperatures for temperatures below $80 \mathrm{~K}$ in $\mathrm{Bi} 2212$, and for temperatures below $40 \mathrm{~K}$ in $\mathrm{Bi} 2223$.

The very similar behavior as a function of AC and DC field in these two high-anisotropy superconductors indicates that the origin of the induced microwave dissipation stems from interaction of $\mathrm{AC}$ field with fluxons that reside between conducting planes.

Although the two compounds exhibit similar behavior, there are some significant differences. The sharp increase of the signal intensity just below $T_{\mathrm{c}}$ in Bi2212 for $H \| a-b$ (Fig. 4) is not observed in the Bi2223 close and below $T_{\mathrm{c}}$ (Fig. 9). Instead, a gradual increase in the intensity with decreasing temperature is followed by a strong anomalous signal at about $85 \mathrm{~K}$ as shown in Figs. 9a and 9b. For $H \| c$ the signal intensity in $\mathrm{Bi} 2212$ at temperatures below the FFR signal is zero down to $4 \mathrm{~K}$, whilst in Bi2223 a strong anomalous signal is observed at about $85 \mathrm{~K}$, very similar to the anomalous signal observed at $H \| a-b$. Our results show that the intensity of the anomalous signal decreases more than two orders of magnitude when increasing the DC field from $0.004 \mathrm{~T}$ to $0.03 \mathrm{~T}$. Thus the interaction of the $\mathrm{AC}$ field with the vortex system below $T_{\mathrm{c}}$ down to $80 \mathrm{~K}$ in $\mathrm{Bi} 2223$ is different than expected assuming that the response below $T_{\mathrm{c}}$ for the two compounds should be similar. However, the two compounds show similar behavior below $80 \mathrm{~K}$, including the sharp increase in the signal intensity in Bi2223 (Fig. 9b)

\section{Comparison between the Experimental Results and Theory}

Based on the present experimental results, the microwave dissipation in layered highly-anisotropic superconductors subjected to a magnetic field parallel to the layers has been studied theoretically [1]. The analysis of the microwave dissipation in the presence of an AC field is based on Josephson electrodynamics in layered type II superconductors. The reader is referred to the article that discusses the microwave dissipation as a function of the DC field and temperature with and without the application of $\mathrm{AC}$ field. It shows that the addition of the low frequency AC magnetic field greatly enhances the microwave signal. It is explained by AC "shaking" effect that depins the Josephson vortices. Thus the theoretical article, referred here as the Theory, and the present experimental work, are complementary. In order to present to the reader a more comprehensive discussion without referring him continuously to the theoretical work, we bring 


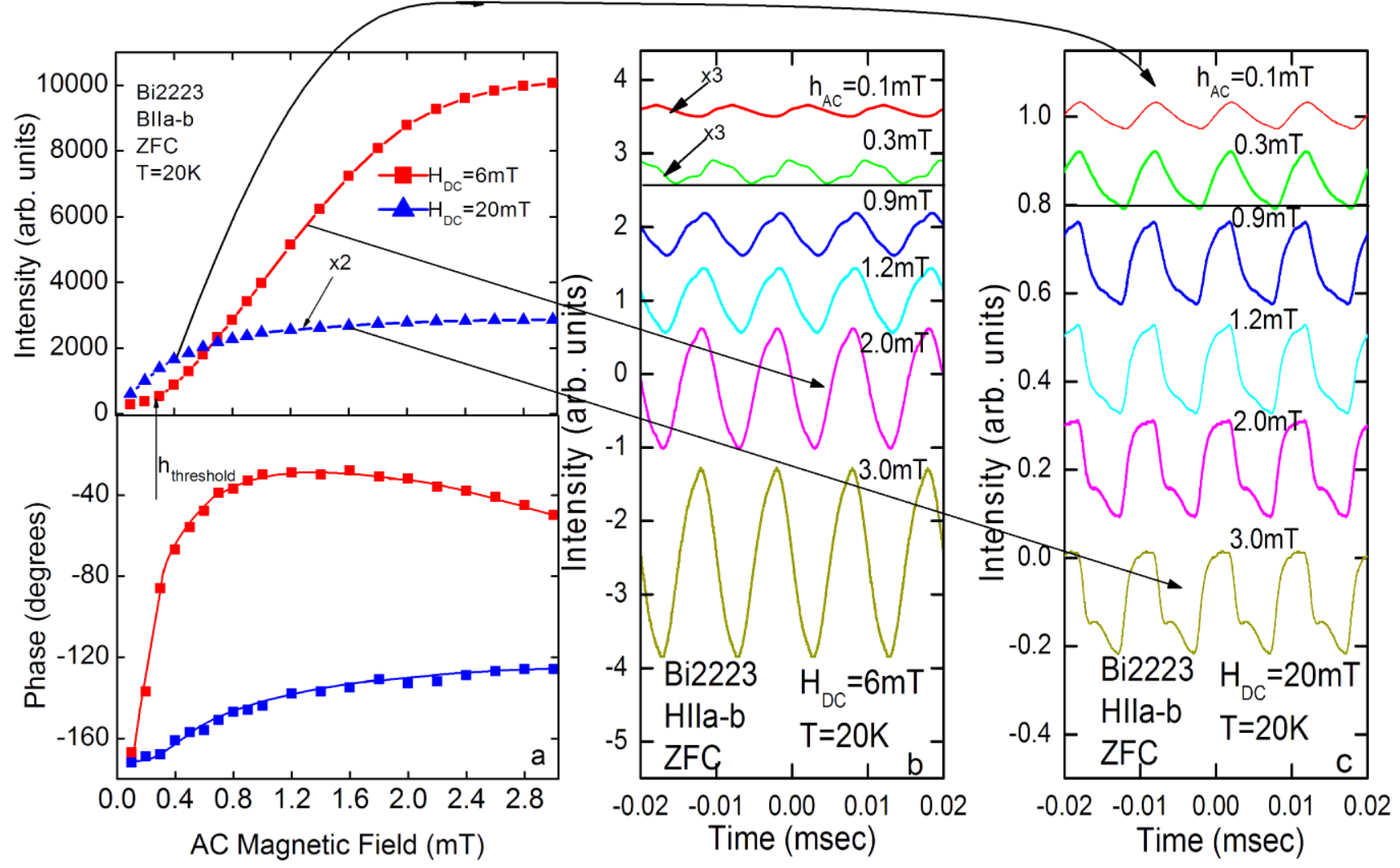

Fig. 11 (a) Lock-in signal intensity and phase in $\mathrm{Bi} 2223$ as a function of AC field at $40 \mathrm{~K}$ at a low-DC field and a high-DC field of $6 \mathrm{mT}$ low-DC field shown in (a). (c) Oscilloscope signal along the curve of and $20 \mathrm{mT}$, respectively. (b) Oscilloscope signal along the curve of the

Fig. 12 Evolution with temperature of the signal intensity as a function of a DC magnetic field in $\mathrm{Bi} 2223$ for $\mathrm{AC}$ fields of $0.3 \mathrm{mT}$ and $1.2 \mathrm{mT}$. The inserts show the variation of the maxima of these signals with temperature

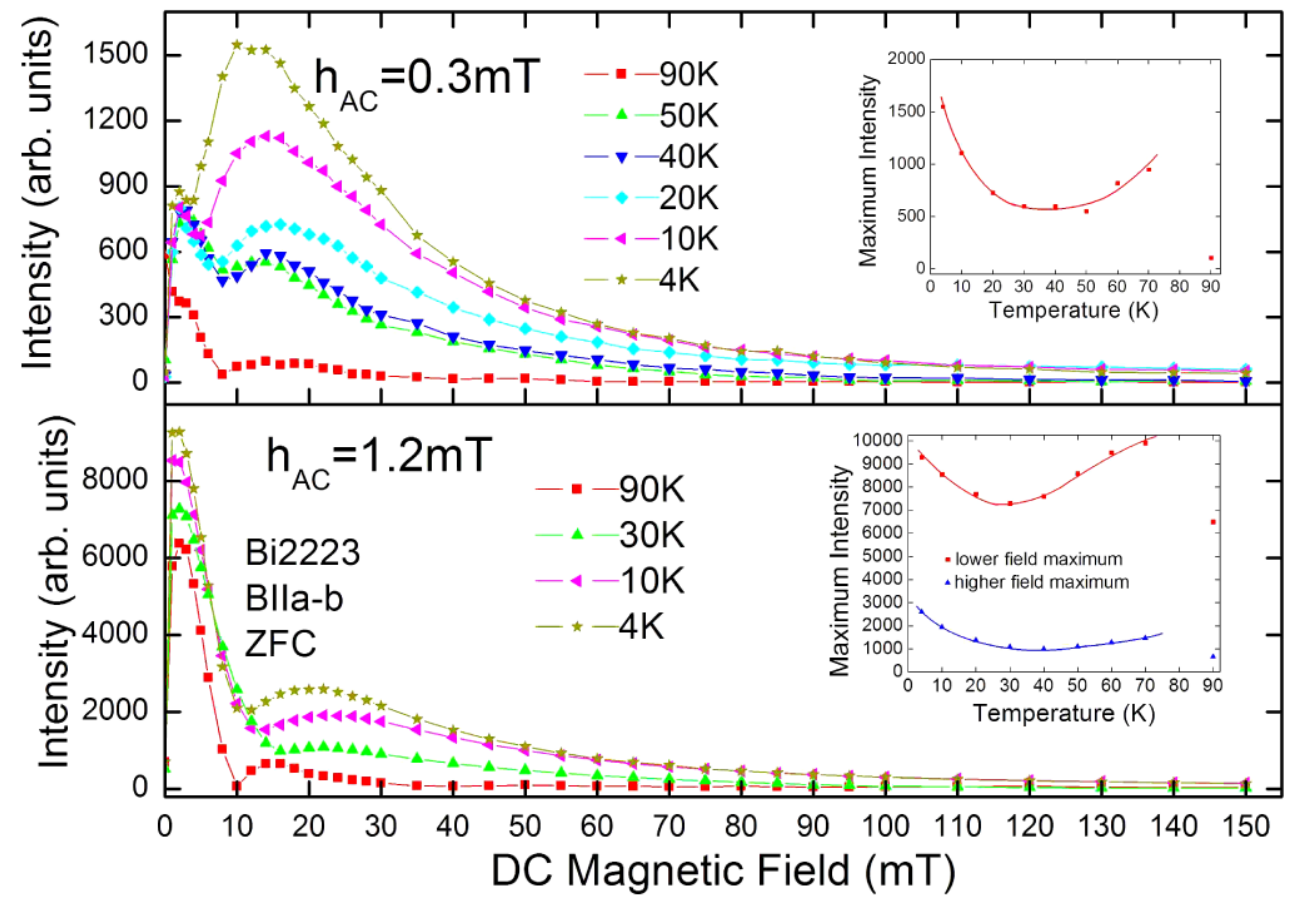




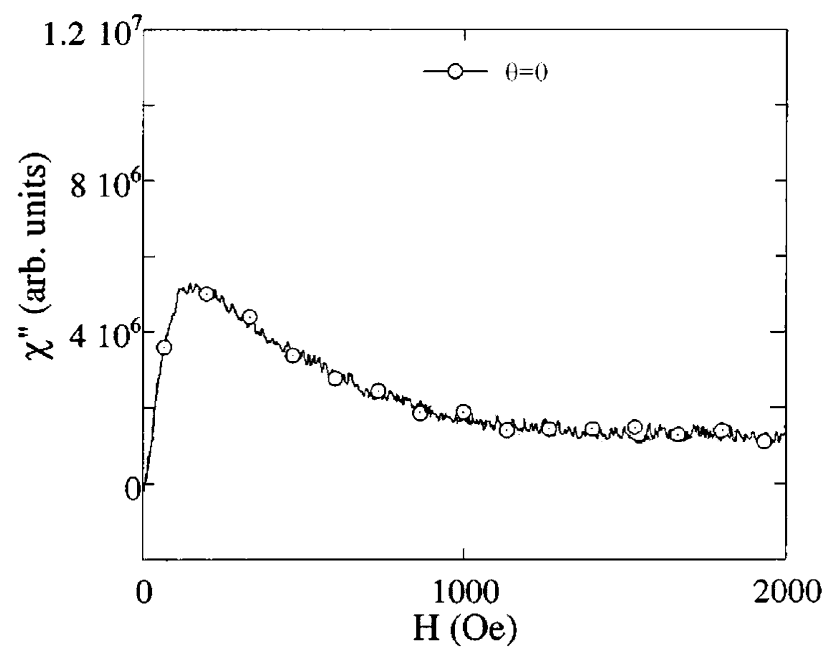

Fig. 13 Bi2212 microwave susceptibility as a function of magnetic field at $70 \mathrm{~K}$ obtained by microwave power modulation and not by of AC magnetic field modulation for $H_{\mathrm{DC}} \| a-b(\theta=0)$ [7]

below the experimental fit to the theoretical results including important conclusions.

The experimental results in the high-anisotropy superconductors Bi2212 and Bi2223 show, in general terms, the presence of two DC magnetic field regions: a low-DC and a high-DC region in which the AC field induces sinusoidal shape signals that are in phase with and out of phase to the AC frequency, respectively. In the small in-between DC field region the signal deviates from the sine form and the phase of the fundamental frequency varies sharply with increasing field, from close to $0^{\circ}$ toward $180^{\circ}$.

The microwave dissipation that results from the interaction of the microwave magnetic field with JV in Bi2212 as a function of a DC magnetic field parallel to the $a-b$ plane, but without the presence of the AC field, has been investigated for DC magnetic field parallel to the $a-b$ plane by Enriquez et al. [7]. The experimental setup was identical to the present experiment using microwave power modulation instead of the magnetic field modulation in the present work. Results of the power dissipation as a function of magnetic field at a temperature of $70 \mathrm{~K}$ without $\mathrm{AC}$ field [7] are shown in Fig. 13. The signal intensity in Enriquez experiment were a few orders of magnitude smaller than those obtained in the present experiment, indicating that the AC field strongly enhances the interaction of the JV with the microwaves in accordance with the shaking effect.

Figures 14, 15 and 16 correspond to Figs. 4a, 5 and 3 of the Theory article [1].

Figure 14 presents a schematic plot of the calculated signal intensity as a function of a DC magnetic field. It shows the presence of two linear regions separated by a nonlinear region in which the phase shifts from $0^{\circ}$ at low field to $180^{\circ}$ at high field. This result is in agreement with the results of Fig. 5 and Fig. 10 for Bi2212 and Bi2223 respectively (at

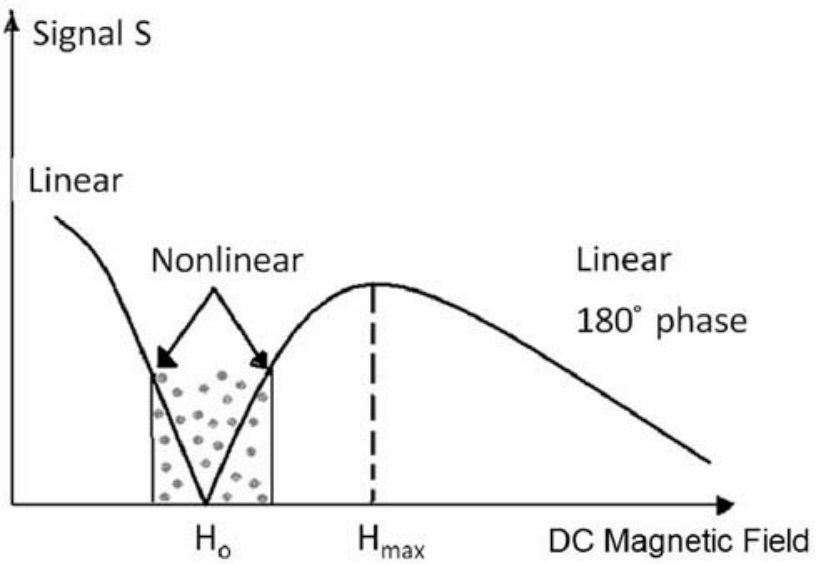

Fig. 14 Schematic plot of a signal intensity as a function of a DC magnetic field in the limit of a vanishing AC field

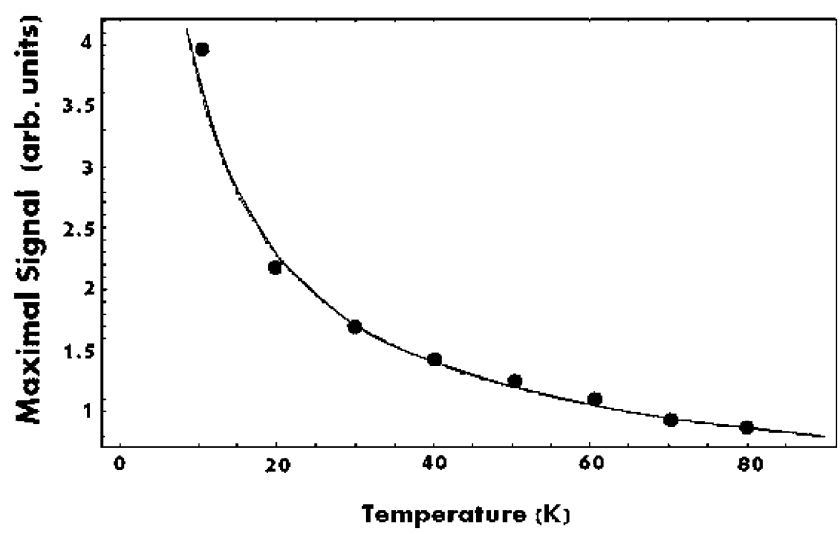

Fig. 15 Fit of the calculated maximum intensity in the signal at temperatures $80 \mathrm{~K}$ to $10 \mathrm{~K}$ with the measured intensities for $h_{\mathrm{AC}}=1.2 \mathrm{mT}$ of Fig. 6

the high-AC field of $1.2 \mathrm{mT}$ ) that show two linear regions whose phase varies from close to $0^{\circ}$, at low-DC fields, to close to $-180^{\circ}$ at high-DC fields, separated by a nonlinear region where the phase shifts continuously toward $-180^{\circ}$.

Figure 15 presents the exponential fit to the maximum signal intensity, $S$, as a function of temperature shown in Fig 6. This exponential fit $S=T^{-\alpha}$ was derived from (42) with $\alpha=0.7$ of the Theory paper.

A remarkable result of the theoretical work was obtained when fitting the experimental results of Enriquez et al. [7] that show the microwave dissipation as a function of DC field in Bi2212 without the application of AC field, Fig. 13. The full circles were fitted in the theoretical work using (36) of the theory article [1]. That infers that the interaction of the JV with the microwave magnetic field with and without the presence of the AC field originates from the same mechanism. The AC field induces the shaking of the JV that strongly enhances the microwave dissipation. 


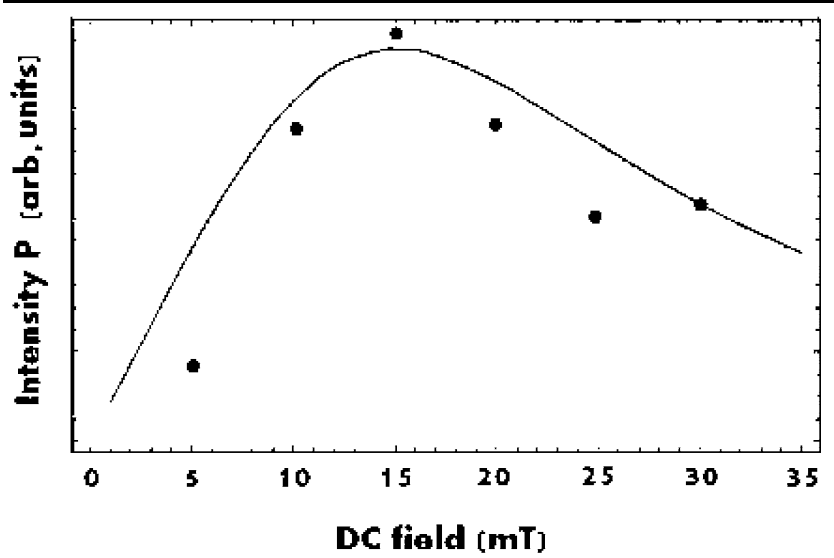

Fig. 16 Theoretical fit to the microwave absorption intensity as a function of $H_{\mathrm{DC}}$, without the presence of the AC field, observed by Enriquez et al. in $\mathrm{Bi} 2212$ [7]

\section{Dependence of Microwave Dissipation on the Microwave Power}

The applied microwave magnetic field in the present work was $0.007 \mathrm{mT}(6.3 \mathrm{~mW}$ in a cavity with $Q=3300)$. The interaction of the JV with the microwave for different microwave power in Fig. 8 shows that the signal intensity for the same AC and DC fields is proportional to the square root of the microwave power implying an ohmic behavior. A similar ohmic behavior was observed by Enriquez [11] using the same experimental setup but without the application of the AC field (see Fig. IV.2.1 in reference [11]). It implies that the JV interaction with the microwave field is not affected by the application of the AC field.

\section{Discussion}

The present work investigates experimentally dynamic properties of JV in the highly anisotropic superconductors $\mathrm{Bi} 2212$ and Bi2223. The JV are generated by applying a DC magnetic field parallel to the conducting plane. Their motion is caused by AC magnetic field collinear to the DC field and detected by microwave dissipation due to interaction of the JV with the microwave magnetic field. Thus three magnetic fields, DC, AC and microwave, affect the intensity of the interaction. In addition, other variables such as temperature, frequency of the AC magnetic field and pinning center can affect the JV interaction with the microwave. Deviation of the magnetic field from the $a-b$ plane [14] introduces PV that impedes the motion of JV. We have shown that small variations of any of the above variables can strongly influence the experimental results. The strong dependence of the observed signal on the large number of parameters made the results complicated to analyze and difficult for the reader, as it is necessary to specify the different parameters when analyzing or presenting the results. Initially the different response of the microwave dissipation as a function of a DC magnetic field with or without the application of an AC field raised difficulties in the analysis of the results. However, the theoretical work presented recently in the theory article has shown that both dissipations stem from the interaction of the JV with the microwave and that the differences in their functional behavior result from the shaking of the JV by the AC field.

Ohmic dissipation of the microwave in the power range $0.2 \mathrm{~mW}$ to $20 \mathrm{~mW}$ with and without and the application of the AC field observed in the present experiment and in Enriquez experiment, respectively, implies that the interaction of the AC magnetic field-but not the microwave magnetic field-is responsible to the observed strong power dissipation. Hence the AC modulating field has an active role in inducing strong power dissipation, in contrast to the passive modulating role observed in electron spin resonance experiments.

The theory article [1] explains the results shown in Figs. 6 and 8 that indicate the presence of two regions at a low-DC and a high-DC fields, in which the dissipation signals differ by $180^{\circ}$ and the response to the AC field is linear. Inbetween these two regions the signal phase shifts from close to $0^{\circ}$ to close to $180^{\circ}$ with a nonlinear signal shape. The strong increase of the microwave dissipation with decreasing temperature shown in Fig. 7 was not observed in microwave dissipation measurements by Enriquez [11] where the AC field was absent. This difference results from the AC field shaking effect, as described in the theory work and presented in Fig. 14.

The response of the signal as a function of DC field for $\mathrm{AC}$ frequencies $0.1 \mathrm{kHz}$ to $100 \mathrm{kHz}$ for the same $\mathrm{AC}$ field was very similar. It indicates that the response in this $A C$ frequency range is not affected by frequency.

The almost identical behavior of the signal intensity in Bi2212 and Bi2223 confirms the theoretical investigation that the microwave dissipation results from the AC-shaking of the JV in high-anisotropy superconducting compounds. It therefore predicts that similar behavior in the microwave dissipation should be observed in all highly anisotropic superconductors. A list of these compounds is discussed by Shimoya et al. [12]. Thus one would expect to observe IMDACMF signals in the under-doped high anisotropy superconductor $\mathrm{YBCO}_{6.6}\left(T_{\mathrm{c}}=60 \mathrm{~K}\right)$ where the coupling between the layers is through the Josephson effect; it has not been observed in the low-anisotropy optimally doped YBCO $\left(T_{\mathrm{c}}=89 \mathrm{~K}\right)$ where there is no Josephson coupling between the layers.

The functional dependence of Bi2212 and Bi2223 at temperatures below $80 \mathrm{~K}$ is similar, in agreement with the theory. Above $80 \mathrm{~K}$ the response to the AC field shows different behavior, including the presence of an anomalous signal in 
Bi2223, observed also at magnetic fields parallel to the $c$ axis. It may indicate the presence of a different JV lattice structure above $80 \mathrm{~K}$ that so far has not been observed by other methods. Figures 6 and 11 show that the signal intensity asa function of the AC field at a high-DC field is saturated above a certain $\mathrm{AC}$ field. The reason for this saturation is not clear. It can be due to the depinning force induced by the AC field. A further investigation is needed.

No AC-induced microwave dissipation is observed for magnetic fields parallel to the c-axis where only PV are formed. The unique property where $\mathrm{AC}$ field induces the shaking of only JV and not PV opens a vast field of investigation where the dynamics of JV exposed to various pinning centers can be investigated. Indeed recently, by using this technique, increase of the interaction between PV and JV obtained by tilting the magnetic field away from the $a-b$ plane has revealed a strong effect of PV on the dynamics of JV [14]. Also this technique has shown a memory effect where a magnetic field can be imprinted into the JV structure [15] and irreversibility behavior of the JV system that results from field cooling procedure [16].

In the present work the AC microwave dissipation has been investigated using an EPR spectrometer at $9.3 \mathrm{GHz}$. EPR spectrometers, whose frequency range is from a few $\mathrm{MHz}$ to hundreds of $\mathrm{GHz}$ have been reported [17]. Applying the technique using EPR spectrometer that operates a different frequencies would provide information on the interaction of fluxons with the electromagnetic field in a very large frequency range. The technique can also be used to investigate interaction of $\mathrm{JV}$ with different defects, including irradiation defects, by comparing the microwave dissipation results before and after introducing the defects. Intense research has been carried out on the dynamic properties of solitons in intrinsic Josephson junction IJJ stacks of highanisotropy superconductors, where the motion of the JV is generated by a DC current parallel to the $c$-axis and detected by I-V curves [18]. It would be interesting to compare the present results with a technique where the motion of the JV is generated by AC current parallel to the $c$-axis and detected via microwave dissipation [19]. It can clarify the difference between the two types of induced motion.

Acknowledgements This work was supported by The Israel Science Foundation Grant No. 499/07, by Heinrich Hertz Minerva Center for High Temperature Superconductivity, by the German Bundesministeriu für Bildung und Forschung BMBF under Contract No. VDI/EKM 139617, by the Deutsche Forschungsgemeinschaft DFG within Sonderforschungsbereich SFB 484 Augsburg, and partly supported by Grant-in-Aid for Scientific Research from the Ministry of Education, Culture, Sports, Science, and Technology, Japan. B.R. is supported by DOE and NSC of R.O.C. Grant No. M93-2112-M009-023. One of us-D.S.-would like to thank O. Shaltiel for analyzing the experimental data.

Open Access This article is distributed under the terms of the Creative Commons Attribution Noncommercial License which permits any noncommercial use, distribution, and reproduction in any medium, provided the original author(s) and source are credited.

\section{References}

1. Shaltiel, D., Krug von Nidda, H.-A., Loidl, A., Rosenstein, B., Shapiro, B.Ya., Shapiro, I., Tamegai, T., Bogoslavski, B.: Phys. Rev. B 77, 014508 (2008)

2. Ustinov, A.V.: Physica D 123, 315 (1998)

3. Clauss, T., Oehmichen, V., Mössle, M., Müller, A., Webber, A., Koelle, D., Kleiner, R.: Supercond. Sci. Technol. 15, 1651 (2002)

4. Hechtficher, G., Kleiner, R., Schlenga, K., Walkenhorst, W., Muller, P.: Phys. Rev. B 55, 14638 (1997)

5. Filatrella, G., Rotoly, G., Grombench-Jensen, N., Pormetier, R.D., Pedersen, N.F.: J. Appl. Phys. 72, 3179 (1992)

6. Shaltiel, D.: J. Low Temp. Phys. 103, 383 (2003)

7. Enriquez, H., Bontemps, N., Foumier, P., Kapitulnik, A., Maignon, A., Ruyter, A.: Phys. Rev. B 53, R14757 (1996)

8. Bohandy, J., Kim, B.F., Adrian, F.J., Moorjani, K., Darcangelis, S., Cowan, D.O.: Phys. Rev. B 43, 3724 (1991)

9. Shaltiel, D., Ginodman, V., Golosovsky, M., Katz, U., Boasson, H., Gerhouser, W., Fisher, P.: Physica C 202, 303 (1992)

10. Tinkham, M.: Phys. Rev. Lett. 61, 1658 (1998)

11. Enriquez, H.: These de doctorat de l'Université de Paris (1997)

12. Shimoya, J., Kitazava, K., Shimizu, K., Ueda, S., Orii, S., Chikumoto, N.: J. Low Temp. Phys. 131(5/6) (2003)

13. Shaltiel, D., Krug von Nidda, H.-A., Loidl, A., Lin, C.T., Liang, B., Kurtz, T., Bogoslavsky, B.: Physica C 433, 93 (2005)

14. Shaltiel, D., Krug von Nidda, H.-A., Shapiro, B.Ya., Rosenstein, B., Loidl, A., Bogoslavsky, B., Shapiro, I., Tamegai, T.: Phys. Rev. B 77, 214522 (2008)

15. Shaltiel, D., Krug von Nida, H.-A., Loidl, A., Tamegai, T., Watanabe, T., Fujii, T., Mattsuda, A.: In: VORTEX V Conference Rhodes (2007)

16. Shaltiel, D., Ioshpe, D., Grayevsky, A., et al.: Phys. Rev. B 36, 4090 (1987)

17. Beeman, B., Fink, H.J., Shaltiel, D.: Phys. Rev. 147, 454 (1966)

18. Yurgens, A.A.: Supercond. Sci. Technol. 13, R85 (2000)

19. Madsen, S., Filatrella, G., Pedersen, N.F.: Eur. Phys. J. B 40, 209 (2004) 